\title{
Erratum to: Optimal Affinity of a Monoclonal Antibody: Guiding Principles Using Mechanistic Modeling
}

\author{
Abhinav Tiwari, ${ }^{1}$ Anson K. Abraham, ${ }^{2}$ John M. Harrold, ${ }^{3}$ Anup Zutshi, ${ }^{4}$ and Pratap Singh ${ }^{1,5}$
}

\section{Erratum to: AAPS J}

\section{DOI: 10.1208/s12248-016-0004-1}

The listing of the affiliation for the second author, Anson K. Abraham, is incorrect in the published article. The company name should be listed as "Merck \& Co., Inc." as shown in this erratum.

The online version of the original article can be found at http:// dx.doi.org/10.1208/s12248-016-0004-1.

\footnotetext{
${ }^{1}$ Pharmacokinetics, Dynamics and Metabolism, Pfizer, Cambridge, Massachusetts, USA.

${ }^{2}$ Pharmacokinetics, Pharmacodynamics and Drug Metabolism, Merck \& Co., Inc., West Point, Pennsylvania, USA.

${ }^{3}$ Department of Pharmacokinetics and Drug Metabolism, Amgen, South San Francisco, California, USA.

${ }^{4}$ EMD Serono, Billerica, Massachusetts, USA.

${ }^{5}$ To whom correspondence should be addressed. (e-mail: pratap.singh.charan@gmail.com)
} 